EUROPEAN ORGANIZATION FOR NUCLEAR RESEARCH

European Laboratory for Particle Physics

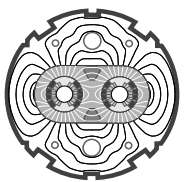

Large Hadron Collider Project

LHC Project Report 102

\title{
SYNCHROTRON RADIATION DOMINATED HADRON COLLIDERS
}

\author{
Eberhard KEIL
}

\begin{abstract}
The synchrotron radiation damping time becomes small compared to the beam lifetime when the beam energy in a circular hadron collider reaches about $100 \mathrm{TeV}$ and the dipole field about 10 T. This paper discusses an approach to the design of these colliders such that the desired performance parameters, e.g. beam-beam tune shift and luminosity are achieved at the equilibrium values of the beam emittance and momentum spread. The design procedure is described, involving the design of the interaction regions, the arcs, and the RF system. The thresholds and/or growth rates of several collective effects, and the growth times for intra-beam scattering are estimated. The consequences of the synchrotron radiation are discussed in the conclusions.
\end{abstract}

Presented at 1997 Particle Accelerator Conference, Vancouver, 12-16 May 1997.

Administrative Secretariat

LHC Division

CERN

$\mathrm{CH}$ - 1211 Geneva 23

Switzerland

Geneva, 3 June 1997 


\title{
SYNCHROTRON RADIATION DOMINATED HADRON COLLIDERS
}

\author{
E. Keil, CERN, Geneva, Switzerland
}

\section{Abstract}

The synchrotron radiation damping time becomes small compared to the beam lifetime when the beam energy in a circular hadron collider reaches about $100 \mathrm{TeV}$ and the dipole field about $10 \mathrm{~T}$. This paper discusses an approach to the design of these colliders such that the desired performance parameters, e.g. beam-beam tune shift and luminosity are achieved at the equilibrium values of the beam emittance and momentum spread. The design procedure is described, involving the design of the interaction regions, the arcs, and the RF system. The thresholds and/or growth rates of several collective effects, and the growth times for intra-beam scattering are estimated. The consequences of the synchrotron radiation are discussed in the conclusions.

\section{INTRODUCTION}

As noticed in the high-field option of really large hadron colliders (RLHC) [1], the damping time $\tau_{z}$ of the oscillations in the three degrees of freedom due to the emission of synchrotron radiation becomes about an hour at a beam energy $E \approx 100 \mathrm{TeV}$ and a dipole field $B \approx 12 \mathrm{~T}$ :

$$
\tau_{z}(h) J_{z}=\frac{16644}{E(\mathrm{TeV}) B(\mathrm{~T})^{2}}\left(\frac{C}{2 \pi \rho}\right)
$$

Here, $z$ labels the plane ( $x$ for horizontal, $y$ for vertical betatron oscillations, $s$ for synchrotron oscillations), $J_{z}$ is the damping partition number, $C$ is the circumference and $\rho$ the bending radius in the arcs of the collider; $C / 2 \pi>R$ includes the long straight insertions for the experiments. Scaling their length like $\sqrt{E}$ from the SSC [2], and assuming four interaction regions, one gets $C / 2 \pi=R+1708 \mathrm{~m}$.

Since the beam lifetime and the duration of a colliding beam run are usually many hours, the beams have the equilibrium beam parameters, given by the equilibrium of quantum excitation and synchrotron radiation damping, during most of their lifetime. Designing an RLHC such that the beam-beam limit and the desired luminosity are achieved at the equilibrium beam parameters has the advantage that the emittances of the injected and circulating beams are decoupled, and that the collider is insensitive to slow phenomena with growth times longer than the damping time.

\section{DESIGN PROCEDURE}

The design procedure for $\mathrm{e}^{+} \mathrm{e}^{-}$colliders [3] in the DESIGN program [4] adjusts the arc and RF system parameters to achieve a specified luminosity. Adapting it to p-p colliders implies setting the mass $m_{p}$, classical radius $r_{p}$, and Compton wavelength $\lambda_{p}$ to their proton values.

\subsection{Interaction Region Design}

For round beams with equal normalised horizontal and vertical emittances $\epsilon_{z}=\gamma \sigma_{z}^{2} / \beta_{z}$, equal $\beta$-functions and vanishing dispersion at the interaction point IP, and ignoring crossing angle and "hourglass" effect, luminosity $L$ and beam-beam tune shift $\xi$ are with bunch population $N$ and collision frequency $f$ :

$$
L=\frac{N^{2} f}{4 \pi \sigma_{x} \sigma_{y}} \quad \xi=\frac{N r_{p}}{4 \pi \epsilon_{n}}
$$

The beams cross at an angle in order to separate them at the parasitic collision points at multiples of half the bunch spacing near the interaction point. Solving (2) for $N f$ yields with the usual relativistic factor $\gamma$ :

$$
N f=L \beta_{x} r_{p} /(\gamma \xi)
$$

The choices of $\beta_{x}=\beta_{y}$ at the IP and of the crossing angle are related [5]. Tab. 1 shows the assumed and resulting IP parameters. The bunch spacing $s$ fixes $f$ by $s=c \beta / f ; c$ is the speed of light. For given $\beta_{x}=\beta_{y}$ and $L, N$ follows from (3), and $\epsilon$ from (2). The beam-beam lifetime $\tau_{b b}=\left(r_{p} / c \sigma_{\text {tot }}\right)\left(\beta_{x} C / \gamma \xi\right)$ is calculated for one interaction region, independent of $L$, proportional to $C$ and $1 / E$, and small for good performance parameters (small $\beta_{x}$ and large $\xi) ; \sigma_{\text {tot }}=120 \mathrm{mb}$ is the total cross section. The small RMS radii $\sigma_{x}=\sigma_{y}$, compared to LHC [6] or SSC [2], are largely due to the higher energy and adiabatic damping.The normalised emittance $\epsilon_{n}$ has about $1 / 3$ of the SSC value.

Table 1: Interaction region parameters for a $100 \mathrm{TeV}$ p-p collider with $\xi_{x}=\xi_{y}=0.003$ [6], $\beta_{x}=\beta_{y}=0.5 \mathrm{~m}$, $s=3.75 \mathrm{~m}$, total inelastic cross section $\sigma_{\text {inel }}=60 \mathrm{mb}$.

\begin{tabular}{lr}
\hline Bunch population $N / 10^{9}$ & 9.03 \\
Normalised emittance $\epsilon_{n} / \mathrm{nm}$ & 367.4 \\
RMS beam radius $\sigma_{x}=\sigma_{y} / \mu \mathrm{m}$ & 1.313 \\
RMS divergence $\sigma_{x}^{\prime}=\sigma_{y}^{\prime} / \mu \mathrm{r}$ & 2.626 \\
Luminosity $L /(\mathrm{nbs})^{-1}$ & 30.1 \\
Events $/$ collision $n_{c}=L \sigma_{\text {inel }} / f$ & 22.6 \\
Beam-beam lifetime $\tau_{b b} / \mathrm{h}$ & 42.4 \\
\hline
\end{tabular}

\subsection{Arc Design}

The arcs should provide just enough quantum excitation to achieve the emittance listed in Tab. 1. The average arc radius $R>\rho$ includes space for the quadrupoles, sextupoles, etc., and short straight sections in the arc cells. In separated 
function FODO cells with $\mu_{x}=\mu_{y}$, normalised horizontal emittance $\epsilon_{n}$, arc tune $Q$ and cell length $L_{p}$ are related by:

$$
\epsilon_{n}=\frac{1}{2} \frac{55 \lambda_{p}}{32 \sqrt{3}} \frac{R F(\mu)}{J_{x} \rho}\left(\frac{\gamma}{Q}\right)^{3} \quad L_{p}=\frac{\mu_{x} R}{Q}
$$

The correction factor for finite phase advances $F(\mu)$ [7] tends towards unity for $\mu_{x}=\mu_{y} \rightarrow 0$. The insertions do not contribute to quantum excitation and momentum compaction $\alpha=(2 \pi R / C) Q^{-2}$. I assume that round beams with $\epsilon_{y} \approx \epsilon_{x}$ are achieved by coupling horizontal and vertical betatron oscillations, and apply a factor $1 / 2$ to the traditional form of (4). Tab. 2 shows the parameters of the arcs. The dipole field shown [8] is needed in order to obtain colliders in the parameter range I wish to study.

Table 2: Parameters of the arcs of a $100 \mathrm{TeV}$ p-p collider with $B=12 \mathrm{~T}, \rho=27.78 \mathrm{~km}$ and $R / \rho=1.25$ [6]

\begin{tabular}{lr}
\hline Arc tune $Q$ & 80.62 \\
Phase advance $\mu_{x} / 2 \pi=\mu_{y} / 2 \pi$ & 0.25 \\
Cell length $L_{p} / \mathrm{m}$ & 677 \\
Maximum amplitude function $\beta_{x} / \mathrm{m}$ & 1156 \\
Maximum dispersion $D_{x} / \mathrm{m}$ & 8.93 \\
\hline
\end{tabular}

The cold vacuum chamber acts as a cryopump. The perforated beam screen absorbs the synchrotron radiation. Its temperature should be much higher than that of the vacuum chamber for good Carnot efficiency. Collective effects, in particular the resistive wall instability (cf. Section 3.1), and the forces during a magnet quench must also be considered.

\subsection{Synchrotron Radiation}

Tab. 3 shows several parameters related to the synchrotron radiation. The total synchrotron radiation power $P$ per beam is three orders of magnitude higher than the $3.6 \mathrm{~kW}$ in the LHC. The normalised power $p=P / 2 \pi R$ is almost two orders of magnitude higher than the $0.2 \mathrm{~W}$ in the LHC. The photon flux $\Phi$, the number of photons per metre of arc and per second, is about half the value in LEP2, operating at $96 \mathrm{GeV}$ and a total current of $14 \mathrm{~mA}$ [9].

Table 3: Synchrotron radiation parameters of a $100 \mathrm{TeV}$ p-p collider with synchrotron radiation loss $U_{s}=28 \mathrm{MeV}$ and horizontal damping time $\tau_{x}=1.5 \mathrm{~h}$.

\begin{tabular}{lr}
\hline Beam current $I / \mathrm{mA}$ & 116 \\
Synchrotron radiation power $P / \mathrm{MW}$ & 3.24 \\
Normalised radiation power $p / \mathrm{Wm}^{-1}$ & 14.8 \\
Critical energy $E_{c} / \mathrm{keV}$ & 12.9 \\
Photon flux $\Phi / 10^{15}(\mathrm{sm})^{-1}$ & 23.3 \\
\hline
\end{tabular}

\subsection{RF System Design}

The values of $U_{s}$ in Tab. 3, $\sigma_{e}$ and $\tau_{q}$ in Tab. 4, and the parameters of the super-conducting RF cavities, i.e. frequency $f_{\mathrm{RF}}=400 \mathrm{MHz}$, unloaded shunt impedance $Z=60.3 \mathrm{G} \Omega / \mathrm{m}$ and filling time $T_{f}=2.4 \mathrm{~s}$, similar to the LHC values, suffice for the design of the RF system. The DESIGN program finds the RF system parameters listed in Tab. $4 ; \sigma_{e}$ and $\sigma_{s}$ are small. However, the bunch area $4 \pi E \sigma_{e} \sigma_{s} / c$, is comparable to those of LHC and SSC.

Table 4: Parameters of the RF system of a $100 \mathrm{TeV}$ p-p collider with circumference $C=228.9 \mathrm{~km}$, harmonic number $h_{\mathrm{RF}}=305415$, and quantum lifetime $\tau_{q}=24 \mathrm{~h}$.

\begin{tabular}{lr}
\hline Voltage $V_{\mathrm{RF}} / \mathrm{MV}$ & 35.9 \\
Relative RMS energy spread $\sigma_{e} / 10^{-6}$ & 6.53 \\
Bucket height $/ 10^{-6}$ & 22.5 \\
Synchrotron tune $Q_{s} / 10^{-3}$ & 1.27 \\
Bunch length $\sigma_{s} / \mathrm{mm}$ & 27.5 \\
Bunch area $4 \pi E \sigma_{e} \sigma_{s} / \mathrm{c} / \mathrm{eVs}$ & 0.754 \\
\hline
\end{tabular}

The time needed for a particle to radiate all its energy, and thus also the time needed for acceleration to the operating energy (when synchrotron radiation is neglected) is one half of $\tau_{x}$. If the acceleration time $T$ is to be shorter than $\tau_{x} / 2$, the peak RF voltage must be higher than that listed in Tab. 4 in the ratio $\tau_{x} /(2 T)$.

\section{COLLECTIVE EFFECTS}

The resistive wall instability, coherent synchrotron tune shift, longitudinal microwave instability, and transverse mode coupling instability TMCI are important [10] for the RLHC [11]. I only study the dominant contribution, and assume injection at $5 \mathrm{TeV}$, and a bunch area $4 \pi E \sigma_{e} \sigma_{s} / c=$ $1 \mathrm{eVs}$ there. Below, $I$ is the total beam current, and $I_{b}$ is the bunch current. The effective impedances $Z_{\text {eff }}$ are the weighted sums of $Z(\omega)$ and the bunch power spectrum [12]. Tab. 5 lists thresholds and growth rates at the energies where they are most critical.The longitudinal microwave and the transverse mode coupling instabilities are below threshold by good factors [13], and not discussed.

\subsection{Transverse Resistive Wall Instability}

The growth rate of the resistive wall instability $\tau_{w}^{-1}$ is [12]:

$$
\tau_{w}^{-1}=\frac{r_{p} \bar{\beta} I F_{w}}{e Z_{0} \gamma b^{3}} \sqrt{\frac{\mu_{0} \rho_{w} C c}{\pi(n-Q)}}
$$

Here, $(n-Q)=0.25$ is the tune of the $n$-th mode, $Z_{0}$ is the impedance of free space, $b$ is the radius of the beam screen, and $\bar{\beta} \approx R / Q$ is the average $\beta$-function. The wall penetration factor $F_{w}=7.17$ describes the effects of a beam screen similar to that in the LHC [6], consisting of a thin inner $\mathrm{Cu}$ layer and a thicker layer of stainless steel. In the LHC, about half the wall resistivity is caused by the $10 \%$ of the circumference with a $\mathrm{Cu}$ vacuum chamber at room temperature. I ignore this factor in the calculation of the growth rates. The growth rate $\tau_{w}^{-1}$ in Tab. 5 corresponds 
to 147 turns, which can be handled by feedback systems. Resistivity and thickness of the inner $\mathrm{Cu}$ layer are critical issues in the design of the beam screen in the LHC [14]. If they are both increased by the same factor, the growth rates and the forces on the screen due to a magnet quench remain constant. This might open the possibility of reducing the cryogenic load of the beam screen by operating at a higher temperature. The resistive power losses due to the image current are much smaller than the synchrotron radiation power.

Table 5: Growth rates and threshold impedances at $100 \mathrm{TeV}$ except where stated otherwise. The beam screen has a radius $b=0.03 \mathrm{~m}$ and consists of two layers. The inner layer is $50 \mu \mathrm{m}$ of $\mathrm{Cu}$ at 5 to $20 \mathrm{~K}$ with a resistivity $\rho_{w}=1.8 \cdot 10^{-10} \Omega \mathrm{m}$, the outer layer is stainless steel.

\begin{tabular}{lr}
\hline Resistive wall growth rate at $5 \mathrm{TeV} \tau_{w}^{-1} / \mathrm{s}^{-1}$ & 8.88 \\
\hline Coh. synchrotron tune shift $\Im\left(Z_{\mathrm{L}} / n\right)_{\text {eff }} / \mathrm{m} \Omega$ & 25.8 \\
Long. $\mu$-wave instability $\left|\left(Z_{\mathrm{L}} / n\right)_{\text {eff }}\right| / \Omega$ & 0.968 \\
TMCI threshold $\Im\left(Z_{\mathrm{T}}\right)_{\text {eff }} / \mathrm{G} \Omega \mathrm{m}^{-1}$ at $5 \mathrm{TeV}$ & 0.575 \\
\hline
\end{tabular}

\subsection{Coherent Synchrotron Tune Shift}

To preserve longitudinal Landau damping, the synchrotron tune shift must remain smaller than the synchrotron tune spread. This leads to an upper limit for the imaginary part of the effective longitudinal impedance [10]:

$$
\Im\left(\frac{Z_{\mathrm{L}}}{n}\right)_{\mathrm{eff}} \leq \frac{6}{\pi^{3}} \frac{h_{\mathrm{RF}}^{3} V_{\mathrm{RF}}}{I_{b}}\left(\frac{2 \pi \sigma_{s}}{C}\right)^{5}
$$

The coherent synchrotron tune shift is driven by the longitudinal broad band impedance that is dominated by the shielded bellows [12] in the LHC. Assuming that the beam screen radius and the number of bellows per unit length are similar to those in the LHC, the effective longitudinal impedance $\Im\left(Z_{\mathrm{L}} / n\right)_{\text {eff }} \approx 0.1 \Omega$ is independent of the machine circumference, and about an order of magnitude higher than the threshold listed in Tab. 5. Increasing the harmonic number $h_{\mathrm{RF}}$ and raising the RF voltage $V_{\mathrm{RF}}$ do not help in overcoming this potential problem. The only promising way of increasing the threshold is increasing $\sigma_{s}$ by a factor $10^{1 / 5} \approx 1.6$, by exciting the synchrotron oscillations with RF noise, by reducing the damping partition number $J_{s}$, or by reducing the slope of the RF wave form with a second RF system at a higher frequency [15]. Since the cut-off due to the bunch length is well above the resonant frequency of the bellows, the shorter bunches than in the LHC should not have much effect on the effective longitudinal impedance.

\subsection{Intra-Beam Scattering}

Intra-beam scattering IBS causes growth of the oscillation amplitudes. The amplitude growth times for synchrotron oscillations $\tau_{s}=1.5 \mathrm{~h}$ and for horizontal betatron oscillations $\tau_{x}=3.2 \mathrm{~h}$, obtained with the actual $\beta$-functions and dispersion in the arc cells, are only about twice the corresponding synchrotron damping times. The vertical intrabeam scattering time is negative, implying damping, and orders of magnitude larger than the other two. In more tightly focused bunches, IBS may become a limitation [13].

\section{CONCLUSIONS}

An inevitable consequence of designing RLHCs to synchrotron radiation damping times of the order of hours is the synchrotron radiation power $P$. When (1) is used to eliminate $B, P$ scales as follows:

$$
P \propto E^{3 / 2} L \beta_{x} \xi^{-1} \tau_{x}^{-1 / 2}
$$

In the same variables, the stored energy $G$ in one beam is:

$$
G=E I C / c e=P \tau_{x} / 2 \propto E^{3 / 2} L \beta_{x} \tau_{x}^{1 / 2} / \xi
$$

Eqs. (7) and (8) demonstrate how $P$ and $G$ are related by $\tau_{x}$ : For a given performance in terms of $E, L, \beta_{x}$ and $\xi$, and large $\tau_{x}, P$ is small and $G$ is large, while for small $\tau_{x}$, $P$ is large and $G$ is small. Numerically, the stored energy in one beam is $G=8.83 \mathrm{GJ}$. This figure should be compared to $0.33 \mathrm{GJ}$ for the LHC [6], and 0.4 GJ for the SSC [2].

Designing RLHCs such that the synchrotron radiation damping time is of the order of an hour and that the desired beam-beam tune shift parameter $\xi=0.003$ and luminosity $L=30(\mathrm{nbs})^{-1}$ are reached at the equilibrium beam parameters leads to interaction region, arc and RF system parameters not too far from extrapolated LHC and SSC parameters. The coherent synchrotron tune shift, driven by the longitudinal broad band impedance of the shielded bellows is above threshold by about an order of magnitude, unless one or the other of the measures listed in Section 3.2 are taken. The IBS growth times are only a factor of two larger than the synchrotron radiation damping times.

\section{REFERENCES}

[1] M.J. Syphers et al., Part. Accel. Conf. (Dallas 1995) 431.

[2] SSC Central Design Group, SSC-SR-2020 (1986).

[3] J. Rees and B. Richter, SLAC Report SPEAR-167 (1973).

[4] DESIGN program written by P.M. Gygi-Hanney.

[5] E. Keil et al., LHC Project Report 88 (CERN, 1997).

[6] The LHC Study Group, CERN/AC/95-05 (LHC) (1995).

[7] H. Wiedemann, PEP Note-303 (1979).

[8] L. Rossi, Proc. 34-th Eloisatron Workshop (Erice, 1996).

[9] The LEP2 Team, CERN-AC/96-01 (LEP2) (1996).

[10] J.T. Rogers, Cornell University CBN 96-14 (1996).

[11] G. Dugan et al., Cornell University CBN 96-18 (1996).

[12] F. Ruggiero, Particle Accelerators 50 (1995) 83.

[13] E. Keil, CERN/SL/97-13 (AP) (1997).

[14] M.M. Karliner et al., Particle Accelerators 50 (1995) 153.

[15] T. Linnecar, private communication (1996). 